\title{
SELF-SUSTAINED OSCILLATION OF GAS-LIQUID FLOW \\ IN A CENTRIFUGAL PUMP WITH SEMI-OPEN IMPELLER
}

\author{
J. KUROKAWA, J. MATSUI, H. TAKADA, and T. HIRAYAMA \\ Yokohama National University \\ 156, Tokiwadai, Hodogaya-ku, Yokohama, 240 Japan
}

\section{Introduction}

When a centrifugal pump is operated at very low discharge with low suction head, the air dissolving in water merges to become bubble and stays inside an impeller channel. The staying bubbles accumulate to form a large bubble zone and decrease pumping head. This phenomena is called "air locking", and often arises when air-rich water is used in a pumping system, such as a water supply system of a building.

The air locking phenomena was also raised when air is supplied forcedly into a suction pipe instead of using air-rich water and low suction head. However, a sever self-sustained oscillation was encountered under the same operating conditions, when a semi-open type impeller was used. In this case the pump had stable head-capacity performance and was operated under non-cavitation condition.

There are many types of self-sustained oscillations caused by gas-liquid flow in pumping system, such as low frequency oscillation accompanying cavitation in [1], [2], the oscillation due to time lag between air inlet and impeller inlet as in [3], and so on. These phenomena is accompanied with severe oscillation of a suction pipe. However, the present oscillation is raised only in a semi-open impeller with no suction pipe oscillation:

In order to elucidate the mechanism of the present oscillation, the instantaneous measurements of blade-to-blade pressure were performed and the parameters influencing the oscillation were determined together with the theoretical considerations.

\section{Nomenclature}

$$
\begin{aligned}
& \text { p. : static pressure } \\
& \rho: \text { density of water } \\
& \mathrm{r}, \mathrm{r}_{2} \text { : radius and impeller radius } \quad \mathrm{U} \text { : impeller tip speed } \\
& Q, q \text { : volume flowrates of water and air, respectively (under suction pressure) } \\
& \mathrm{x} \text { : distance along vane center line from leading edge } \\
& \phi \text { : discharge coefficient, defined as } Q /\left(A_{2} U\right) \text { ( } A_{2} \text { : impeller outlet area) } \\
& \psi \text { : head coefficient, defined as } \mathrm{H} /\left(\mathrm{U}^{2} / 2 \mathrm{~g}\right) \text { ( } \mathrm{H} \text { : pump head) } \\
& \psi_{s}: \text { coefficient of static pressure, defined as }\left(p-p_{s 1}\right) /\left(\rho U^{2} / 2\right) \\
& \tau \text { : power coefficient, defined as } \mathrm{P}_{w} /\left(\rho \mathrm{A}_{2} \mathrm{U}^{3} / 2\right)\left(\mathrm{P}_{\mathrm{w}} \text { : shaft power }\right) \\
& \text { subscripts s1, s2: } 900 \mathrm{~mm} \text { and } 16 \mathrm{~mm} \text { upstream of impeller inlet, respectively } \\
& \text { d : discharge ( } 400 \mathrm{~mm} \text { downstream of impeller outlet) }
\end{aligned}
$$




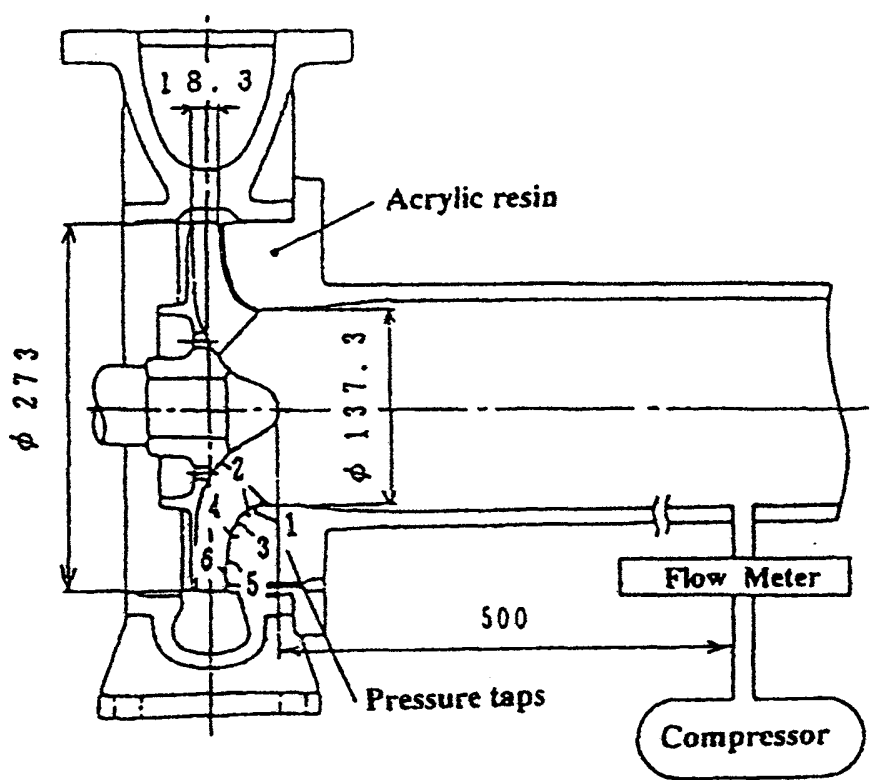

Figure 1. Test pump

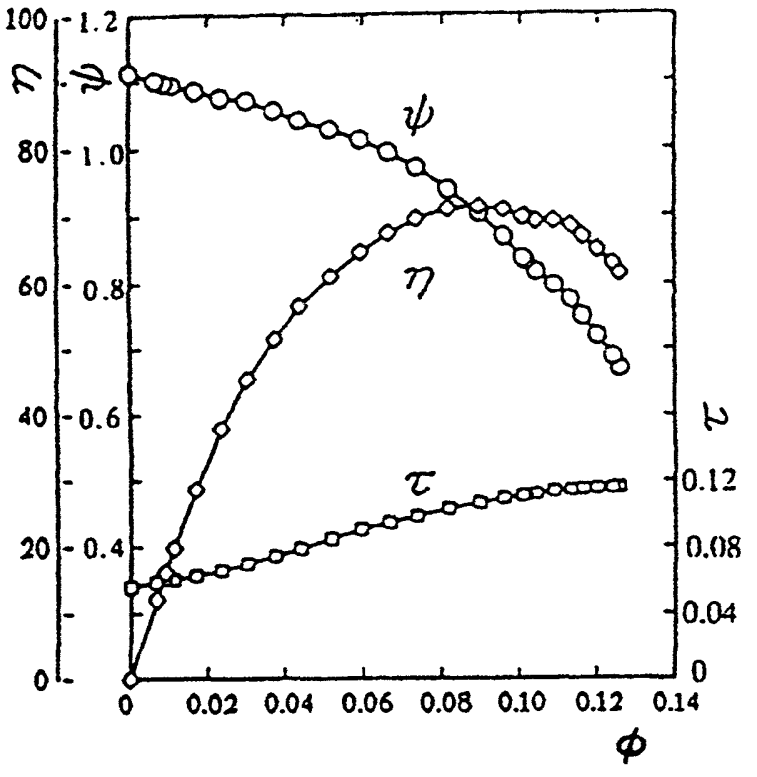

Figure 2. Steady characteristic curves of pump

\section{Experimental Apparatus and Dimensions}

The pump tested is shown in Fig. 1. The impeller is a semi-open type, of which specific speed is $230\left[\mathrm{~m}, \mathrm{~m}^{3} / \mathrm{min}\right.$, rpm] , blade outlet angle $\beta_{2}=25^{\circ}$, radius $\mathrm{r}_{2}=137 \mathrm{~mm}$, blade number 5 , and tip clearance $1 \mathrm{~mm}$. The suction cover and the discharge pipe are made of acrylic resin so that the bubble behavior can be observed. On the suction cover six pressure holes, No. 1 6 shown in Fig 1, are drilled and semi-conductor type pressure transducers (natural frequency of $10 \mathrm{kHz}$ ) are mounted for instantaneous measurements.

The test apparatus consists of a suction pipe of $2 \mathrm{~m}$ length, a pump, a discharge pipe of $10 \mathrm{~m}$ length and a reservoir. The compressed air is supplied at $500 \mathrm{~mm}$ upstream of the impeller inlet, and is separated in the reservoir. A swirl stop is mounted at $600 \mathrm{~mm}$ upstream of the impeller inlet. The pump rotational speed was varied from $500 \mathrm{rpm}$ to $1800 \mathrm{rpm}, \mathrm{corresponding} \mathrm{Reynolds} \mathrm{number}$ ranging from $9.8 \times 10^{5}$ to $3.5 \times 10^{6}$.

\section{Experimental Results and Discussions}

\section{1 PUMP PERFORMANCE}

The pump tested has stable characteristic curves as shown in Fig. 2 with a negative gradient of head-capacity curve over the whole operating range.

When the pump is operated at very low discharge and air is supplied into a suction pipe, the pumping head gradually decreases and begins to oscillate, as shown in Fig. 3. Not only pump head $\psi$ but also discharge coefficient $\phi$, discharge pressure

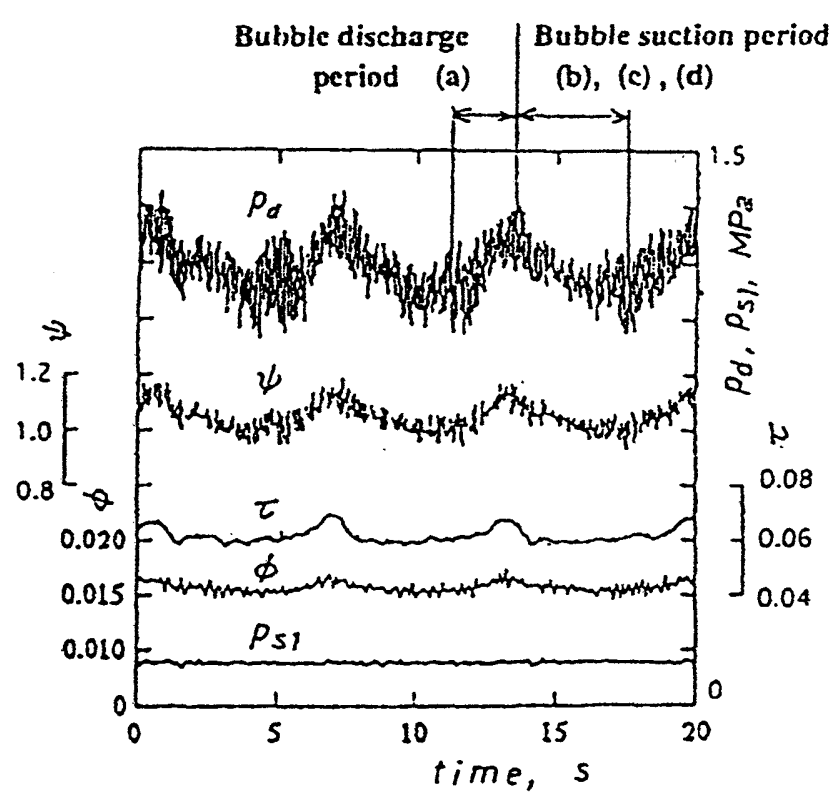

Figure 3. Oscillation of pumping head and others $(Q / q=0.014)$ 


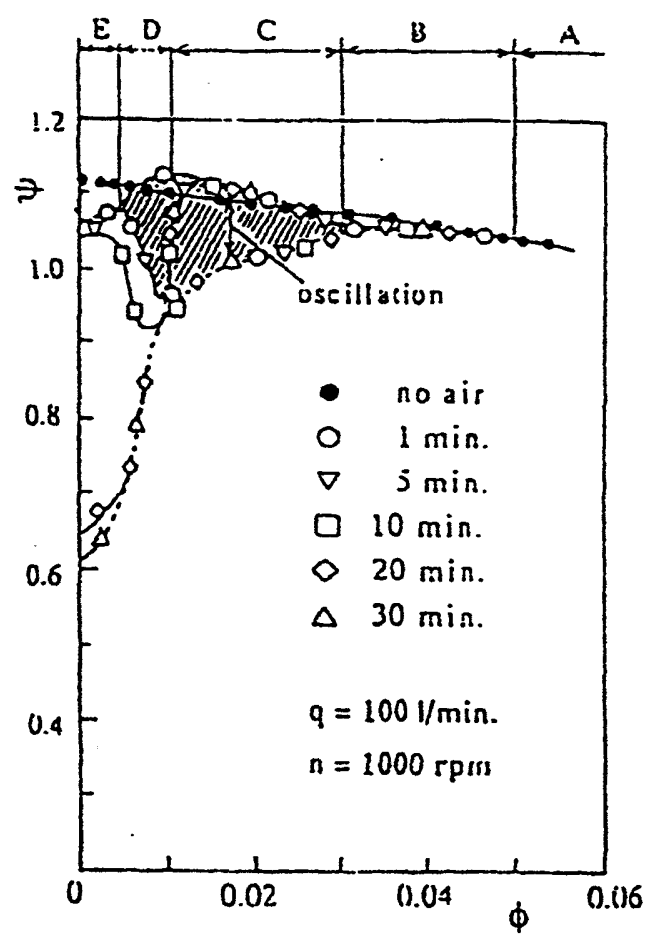

Figure 4. Change in head curve with time

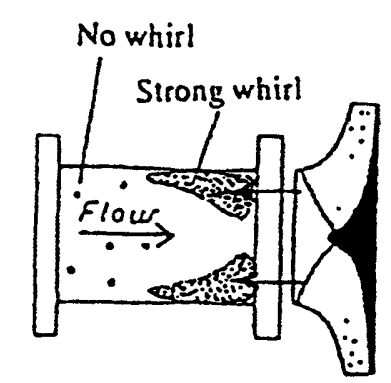

Bubble discharge period (a) (b)

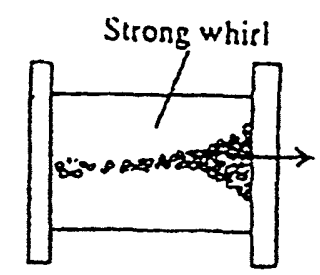

(c)

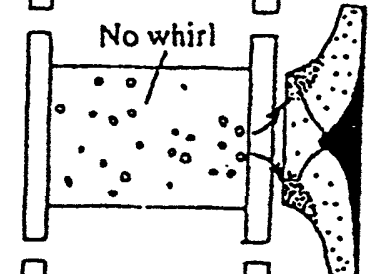

(d)

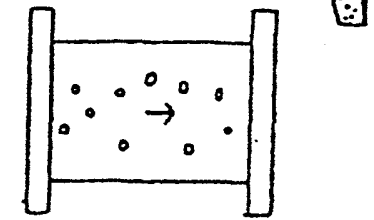

Bubble suction period

Figure 5. Sketch of bubble behavior in a suction pipe while oscillating (Pattern C)

$p_{d}$ and shaft power coefficient $\tau$ oscillate with the period of $6.7 \mathrm{~s}$ and in the same phase. However, the pressure $\mathrm{p}_{\mathrm{s} 1}$ at $900 \mathrm{~mm}$ upstream of the impeller inlet does not oscillate.

Time dependence of head curve is shown in Fig. 4. Both the maximum and the minimum values are plotted when oscillation occurs. After 30 minutes from the start of air supply, the oscillation is still maintained only in the discharge range $C$ shown in Fig. 4 , and in the other range the head curve comes to be the dotted line showing a steady operation. From Fig. 4 it is recognized that there exists following five patterns ;

A: Pump operates steadily without decrease in pumping head, though a bubble zone exists in an impeller channel and many small bubbles discharge at the pump outlet.

$B$; Flow pattern is almost same as $A$, but the blade channel is full of small bubbles, the pumping head slightly decreases and the head-capacity curve becomes horizontal.

C; Sever oscillation occurs with periodical bubble discharge from the impeller inlet to the upstream suction pipe. The oscillation becomes larger with a decrease in pump discharge. Pressure fluctuation of this pattern is shown in Fig. 3 and the sketch of the flow behavior is illustrated in Fig. 5 .

The bubbles accumulating in the impeller are suddenly discharged to the upstream along the casing wall with strong whirl, and liquid single flow enters into the impeller in the pipe center (Fig. 5(a)). Many small bubbles spreading in the pipe then gather to the pipe center due to a strong whirl, grow to large bubbles, form a large group of bubbles (Fig. $5(\mathrm{~b})$ ) and then come into the impeller. As the inlet whirl then gradually disappears, the residual bubbles spread to a whole pipe and come into the impeller together with those from the upstream (Fig. 5(c), (d)). The flow pattern again returns to Fig. $5(\mathrm{a})$.

Throughout one cycle of oscillation, small bubbles flows out steadily at the pump outlet, though the discharge pressure $p_{d}$ fluctuates largely as shown in Fig. 3.

$D$; The impeller inlet flow is separated to liquid flow and gas flow. Soon after the start of air supply the oscillation occurs between two curves shown in Fig. 4, but after 


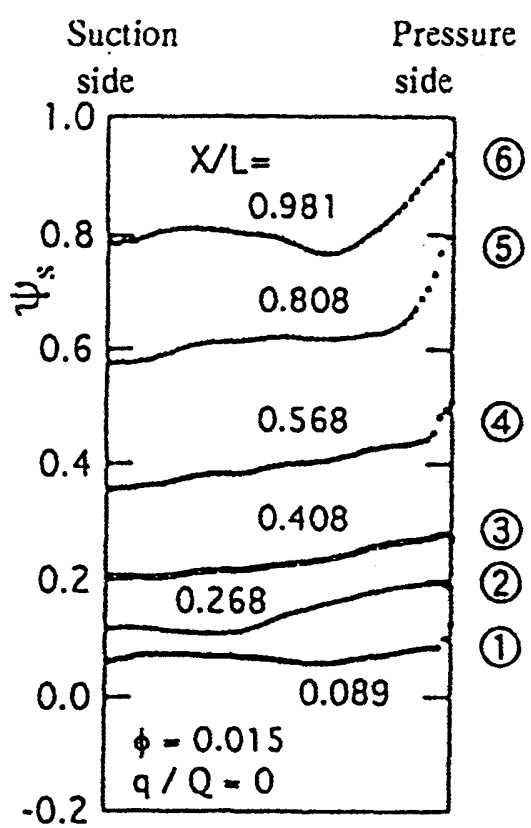

(a) No bubble case

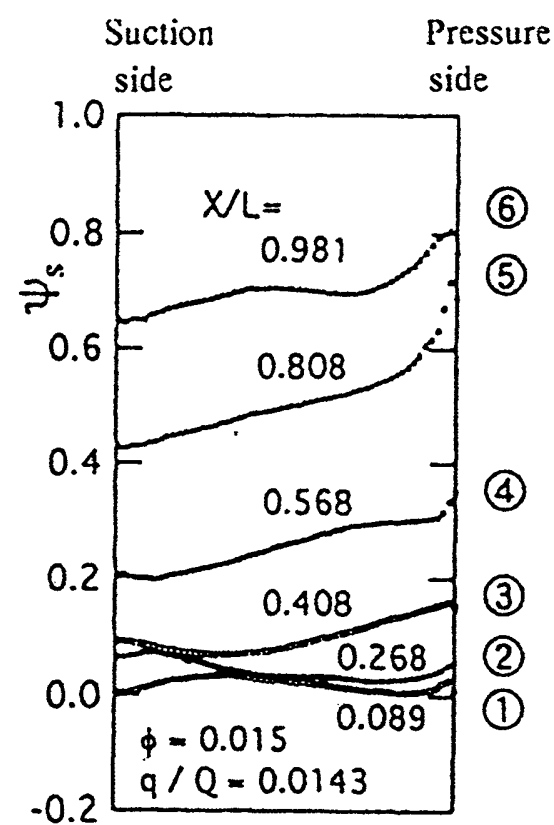

(b) Bubble supply case

Figure 6. Blade-to-blade pressure distribution ( $\phi=0.015,1000 \mathrm{rpm})$

5 minutes it ceases and the head curve comes to the dotted line. The amplitude of oscillation decreases with a decrease in pump discharge.

E; Bubbles accumulate in the impeller inlet region without oscillation, and spread to occupy the upper part of suction pipe, which decreases the pumping head gradually.

\section{2 INSTANTANEOUS BLADE-TO-BLADE PRESSURE DISTRIBUTIONS}

In the case of sever oscillation (pattern C), the shaft power also oscillates, which implies that the blade loading fluctuates. The instantaneous pressure of one impeller channel is then measured and was averaged over 50 rotations at each measuring point. The averaged values of 50-rotations didn't show significant difference from those of 200-rotations. The blade-to-blade pressure distributions thus obtained are shown in Fig. 6, in which the liquid single flow case in Fig. 6(a) is compared with the gas-liquid flow case in Fig. 6(b).

Comparison of Figs. 6(a) and $6(\mathrm{~b})$ reveals that air supply decreases the pressure in the whole impeller channel, when the oscillation occurs. A remarkable difference caused by air supply is seen in the measuring points (1), (2)and(3). This shows that bubbles accumulate around the impeller inlet region and decreases the blade loading. However, the pressure at (5) is seen to recover largely, which shows that the blade loading near the outlet becomes larger than that in the no air case.

It is then concluded that the head drop due to air supply is caused by the loss of blade loading around the impeller inlet due to the accumulation of bubbles.

\subsection{Bubble discharge period and bubble suction period}

The above-described results are obtained by the 50-rotations-averaged values, corresponding to 3 cycles of oscillation, but the instantaneous blade-to-blade pressure distribution must be different from the averaged one especially in the bubble accumulating period. The data averaging period is thus divided to the bubble discharge period and the bubble 


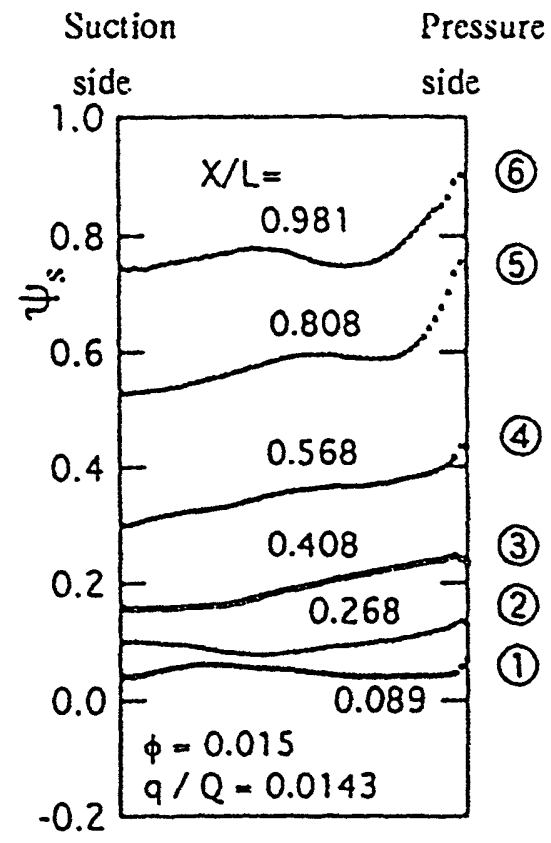

(a) Bubble discharge period

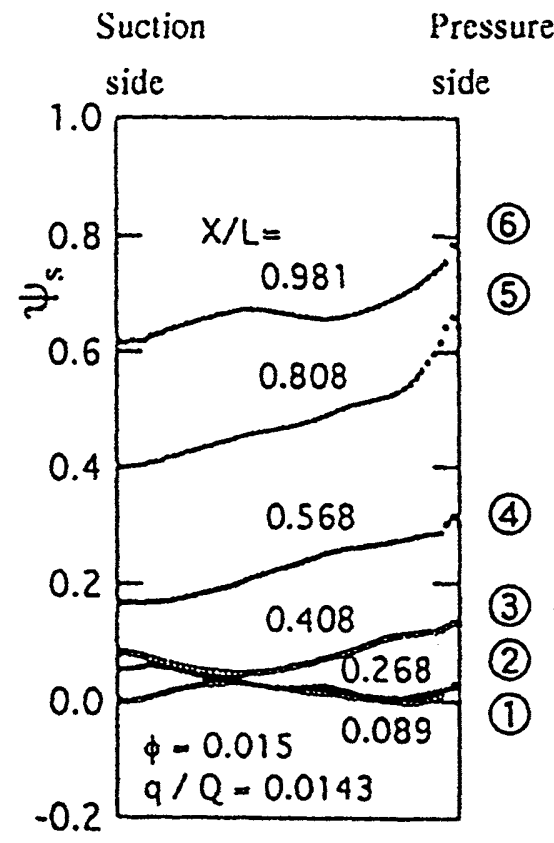

(b) Bubble suction period

Figure 7. Comparison of instantaneous blade-to-blade pressure distribution $(\phi=0.015,1000 \mathrm{rpm})$

suction period by manually switching the data acquisition with observing the phenomena, and the results are compared in Figs. 7(a) and (b).

Comparison of Figs. 7 with Fig. 6 reveals that the instantaneous blade-to-blade pressure profile at bubble discharge period (Fig. 7(a)) is almost same as that of no air case (Fig. 6(a)) and that at bubble suction period (Fig. 7(b)) is almost same as that of the one-cycle averaged (Fig. 6(b)). That is to say, the bubble suction period occupies large part of one cycle of oscillation, and after that bubbles are rapidly discharged to the upstream. In the latter case the impeller is filled only with liquid and attains the maximum pumping head which is equal to that of no air case.

\subsection{Influence of Air Flowrate on Blade-to-Blade Pressure Distribution}

When the air flowrate ratio is increased, the instantaneous pressure distribution between blades changes as shown in Fig. 8, of which lower figures show the net pressure rise along a blade obtained by subtracting the pressure rise due to centrifugal force.

The upper figures reveal that the pressure decrease is considerable especially around the leading edge, and that the pressures at (1), (2) and (3) decreases in order with an increase in air flowrate ratio, while the pressure at (6) changes little. This shows that the bubble accumulation zone gradually spreads to the downstream from the leading edge.

In the lower figures in Fig. 8, the pressure difference between pressure side and suction side expresses the blade loading and decreases rapidly in the upstream half while increases in the downstream half with an increase in air flowrate ratio.

Comparison of Figs. 8(a) with 8(b) (d) also reveals that the air supply decreases the pressure especially in the blade pressure side. Visual observation revealed that bubbles accumulate much more in the pressure side. These two results reveals that the bubbles accumulating in the pressure side near the leading edge decreases the blade loading and makes the main flow deviate to the blade suction side. The deviation of main flow should decrease the pressure recovery in the suction side and the pressure at the downstream 

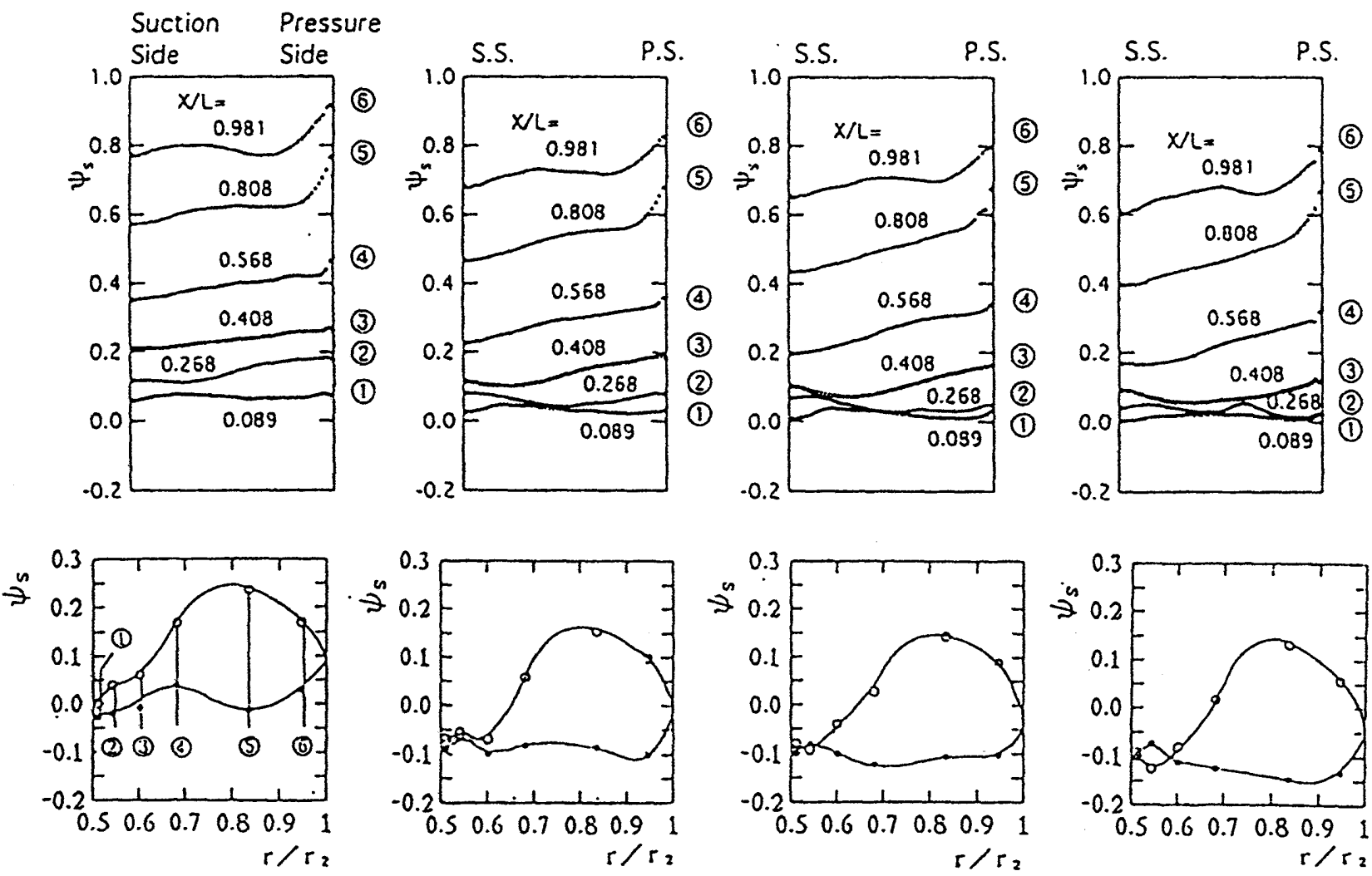

(a) $q / Q=0$

(b) $q / Q=0.0072$

(c) $q / Q=0.0143$

(c) $q / Q=0.0286$

Figure 8. Change in instantaneous blade-to-blade pressure distribution by the change of air flowrate ratio

$$
(\phi=0.015,1000 \mathrm{rpm})
$$

of bubble accumulation zone should recover rapidly due to sudden expansion of main flow, as is shown in Fig. 7. Thus the bubbles accumulate mainly in the blade pressure side at very low discharge, though they accumulate in the suction side at BEP as in [4].

Though sever oscillation is induced at impeller inlet region, the pressure $\mathrm{p}_{\mathrm{s} 1}$ at 900 $\mathrm{mm}$ upstream of the impeller is kept constant as shown in Fig. 3. This suggests that the flow oscillation is limited to the pump inlet region and influence little to the upstream suction pipe. To make this point clearer, the variation of pressure $\mathrm{p}_{s 2}$ at $50 \mathrm{~mm}$ upstream of the impeller inlet is shown in Fig. 9. The pressure $p_{s 2}$ is seen to oscillate in the same phase with the maximum value equal to that of no-air case, the minimum value equal to the upstream pressure $p_{s 1}$. This pressure fluctuation is due to a strong periodical whirl of the recirculation flow which exists near the suction pipe wall of pump inlet.

\section{3 CHARACTERISTICS OF PRESENT OSCILLATION}

The period and the amplitude of present oscillation depend largely on the air flowrate and the pump rotational speed. In order to reveal the characteristics of the present oscillation, the variations of amplitude and frequency are shown in Figs. 10 (a) and (b), when the pump rotational speed and the flowrate ratio $q / Q$ of air to liquid are varied.

When the air ratio is increased under the same rotational speed, the amplitude ratio $\Delta \psi / \psi$ of pumping head becomes larger and the frequency becomes smaller. In this case the visual observation revealed that the bubble accumulation zone along a blade becomes larger and both the scale and the strength of oscillation become larger with an increase in air flowrate ratio. When the pump rotational speed is increased, both the amplitude and 


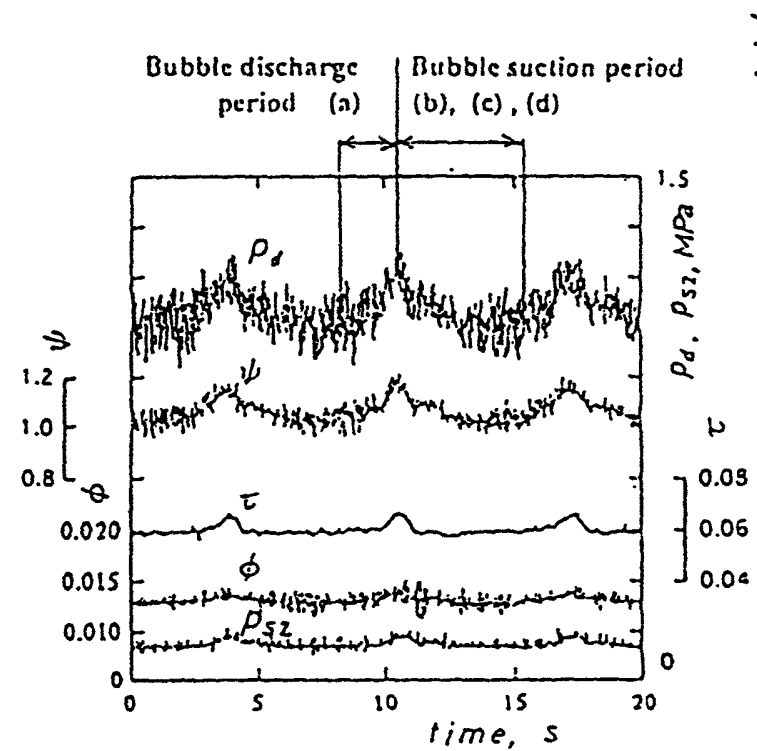

Figure 9. Fluctuation of $\mathrm{p}_{\mathrm{s} 2}$

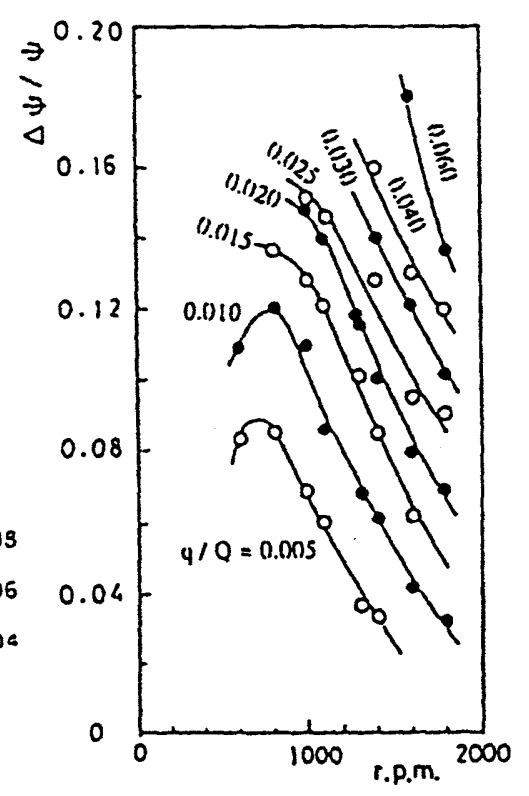

(a) Amplitude ratio $\Delta \psi / / \psi$

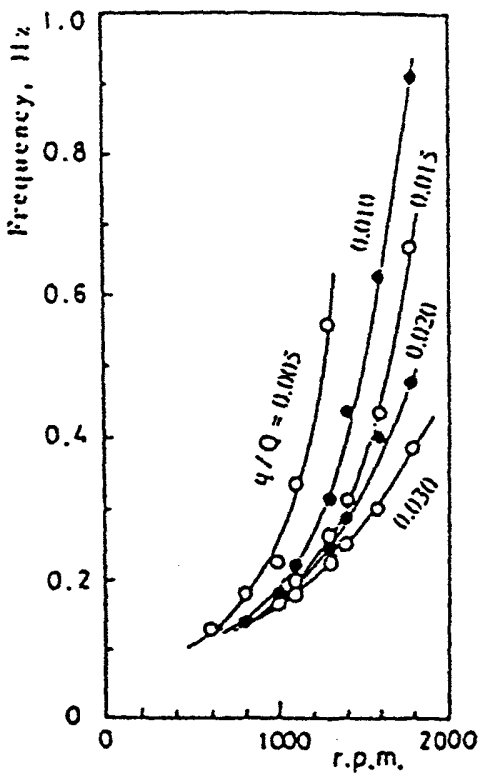

(b) Frequency

Figure 10. Influence of rotational speed and air flowrate ratio

the frequency become smaller resulting in smaller-scaled oscillation.

The present oscillation is one of the unstable phenomenon accompanying gas-liquid flow in a pumping system. The characteristics of the present oscillation is that it occurs in a very low discharge range(10 30\% of the designed) in which large recirculation flow should be induced if there were no oscillation, and that it occurs only by supplying more than only $0.5 \%$ of air to liquid. The length of recirculation flow zone in a suction pipe is then considered to influence the phenomena, but the removal of the swirl stop at $600 \mathrm{~mm}$ upstream in the suction pipe revealed no significant change in the phenomena.

Another remarkable characteristics of the present oscillation is that it occurs only in a semi-open type impeller and doesn't occur in a closed type impeller. The tip clearance was thus considered to influence the phenomena, but the zero clearance test by attaching a rubber plate at the impeller tip revealed no significant change in the phenomena.

\section{Theoretical Considerations on the Mechanism of Present Oscillation}

In order to elucidate the mechanism and the cause of the present oscillation, the stability: analysis based on Gleitzer [5] is applied to the present pumping system.

In the pumping system shown in Fig. 11(a) under non-cavitation condition, it is assumed that the fluctuations of pressure $p$, liquid flowrate $q$ and gas flowrate $q_{G}$ (these notations are different from the preceding chapters) be small compared with the mean pressure $P$ and the mean flowrates $Q, Q_{C}$ of liquid and gas, respectively. Under these assumptions a perturbation method can be introduced to linearize the equation. It is also assumed that the air flowrate at pump outlet is constant and subscripts 1 and 2 are given to the pump suction side and the discharge side, respectively.

Considering that the bubble flow rate is small compared with the liquid flowrate, and that the valve resistance is dominating in the discharge pipe, the linear equations of motion as for the fluctuation terms in the suction pipe, the discharge pipe and the pump are expressed as follows in the non-dimensional form; 


$$
\begin{gathered}
p_{1}=-L \dot{q}_{1} \\
p_{2}=R v q_{2} \\
p_{2}-p_{1}=R_{p} q_{1}-\dot{q}_{1}+R_{G} q_{G}
\end{gathered}
$$

Here, the pressure $p$ is normalized by $\rho U^{2} / 2$, the flowrate by $A U$ and the time $t$ by $2 L_{p}$ l $U$, where $U, A$ and $L_{p}$ are the impeller tip speed, the sectional area of pipe and the equivalent length of pump, respectively. The notation $\dot{q}_{1}$ denotes the differentiation of $q_{1}$ as for time $t . \quad L$ is the length of the inlet recirculation zone (or the length of suction pipe if there is no reverse flow) normalized by $L_{p}$. The parameters $R_{p}$, $R v$ and $R_{G}$ are the gradients of the characteristic curves of the pump system shown in Figs. 11 (b) and (c), and are defined as

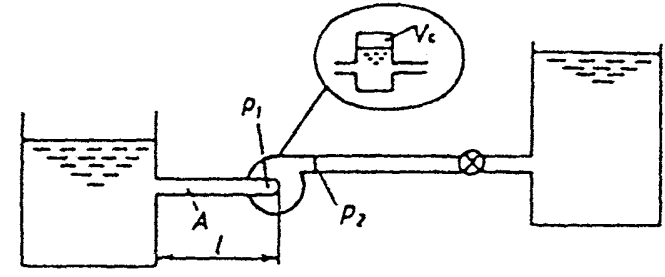

(a) Pump system
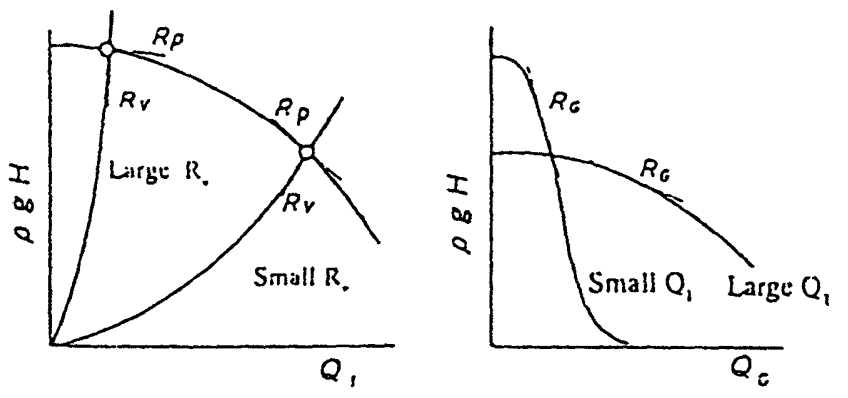

(b) $H-Q_{1}$ curve

(c) $H-Q_{G}$ curve $\left(Q_{1}=\right.$ const. $)$

Figure 11. Model and characteristic curves of pumping system and system resistance

$$
R_{p} \equiv d\left(2 g H / U^{2}\right) / d\left(Q_{1} / A U\right), \quad R \nu \equiv d\left(2 g H v / U^{2}\right) / d\left(Q_{1} / A U\right), R_{G} \equiv d\left(2 g H / U^{2}\right) / d\left(Q_{G} / A U\right)
$$

where $H$ and $H \nu$ are the pumping head and the system resistance(valve), respectively.

Putting the air volume accumulating in an impeller as $V_{c}$ and its fluctuation as $\delta V_{c}$ normalized by $A L_{p}$, the following equations are obtained in the non-dimensional form;

$$
\begin{aligned}
\dot{V}_{c} & =q_{2}-q_{1}+q_{G} \\
\delta V_{c} & =C_{P} p_{1}+M_{b} q_{1}+M_{G} q_{G}
\end{aligned}
$$

where $C_{p} \equiv \partial V_{c} / \partial_{p_{1}}, M_{b}=\partial V_{c} / \partial Q_{1}, M_{G} \equiv \partial V_{c} / \partial Q_{G}$

The motion of a bubble is to be determined from the balance of forces acting on a bubble. Here for the simplicity, the fluctuation of the bubble volume at an impeller inlet is assumed as follows in the non-dimensional form;

$$
q_{G}=\alpha p_{1}+\beta q_{1}
$$

where $\alpha \equiv \partial Q_{G} / \partial P_{1}, \beta \equiv \partial Q_{G} / \partial Q_{1}$

Equations (1) to (6) are all linear with constant coefficients. Hence the solutions are of the form $e^{\text {st }}$. For stability the real part of $s$ must be negative. Substituting expressions of the form $e^{\text {st }}$ for the perturbations, the growth rate must satisfy the following equation, if there are to be non-tribial solutions to the resulting sy'stem;

$$
s^{2}+a s+b=0
$$

where $a=-\left\{a+\left(M_{b}+\beta M_{c}\right) / L+\left(1+1 / L+\alpha R_{c}\right) / R_{V}\right\} /\left(c_{p}+\alpha_{M_{G}}\right)$

$$
b=-\left\{1-\beta-\left(R_{p}+\beta R_{G}\right) / R_{V}\right\} / L\left(c_{p}+\alpha_{M_{G}}\right)
$$

Generally speaking, the negative valve of $b$ gives static instability, while the 
negative value of $a$ gives dynamic instability if $b$ is positive. In the present pumping system the parameters in the above-equations are considered to be as follows;

$$
C_{P}<0, M_{b}<0, M_{G}>0, R_{p}<0, R_{v}>0, R_{G}<0, \quad \alpha<0, \beta \sim 0
$$

Thus the denominators of $a$ and $b$ are negative and $b$ takes a positive value, but the quantity $a$ can be positive or negative depending on the numerator of $a$. After all for the dynamic instability must satisfy the following equation.

$$
\alpha+M_{b} / L+\left(1+1 / L+\alpha R_{G}\right) / R_{v}<0
$$

The first two terms are negative, and the last term in bracket is positive. The self-sustained oscillation could thus be caused depending on these parameters. If the liquid flowrate is large, the system resistance $R_{v}$, becomes small, resulting that the sy'stem is stable. With an increase in system resistance, the flowrate decreases and the last term becomes very small, which satisfies Eq. (8), resulting in an unstable system. When the flowrate ratio of air to liquid is large, the value $\alpha R_{G}$ becomes large, resulting in dynamic stability.

From the above-described theoretical considerations, it is concluded that the present self-sustained oscillation could be caused by the unbalance between the system resistance rate $R_{V}$ and the loss rate of blade loading $R_{G}$ under the influenced of the behavior $M_{b}$ of the bubble accumulating zone.

One of the other remarkable characteristics is that the oscillation occurs only in a semi-open type impeller. The careful observation revealed that the bubbles in an impeller channel become very small-sized, and accumulate mainly near the blade pressure side. This might be caused by the existence of recirculating flow zone induced by flow separation of blade suction side, as shown by the three-dimensional structurein in Fig.13(a). When the bubble zone further grows, the recirculation flow zone might be suppressed and disappear,because the recirculation flow of a semi-open type impeller is very unstable due to the lack of the outer shroud wall.

The visualization of flow behavior using tufts attached on casing wall revealed that the recirculation flow disappears instantaneously and at the same time the bubble accumulating zone rapidly grows to the upstream, and that the casing wall just in front of the impeller inlet is covered with air layer rotating much more slowly than the impeller.

When inlet recirculation flow disappears, both the meridional and the tangential velocities rapidly decreases at an impeller inlet, which results in large angle of attack at blade inlet. A large-sized recirculation flow is thus induced again, and the bubbles are suddenly discharged to the upstream with the recirculation flow. During the period from bubble discharge to bubble gathering to the pipe center, the liquid flowrate increases at impeller inlet, and the pumping head increases due to the recovery of

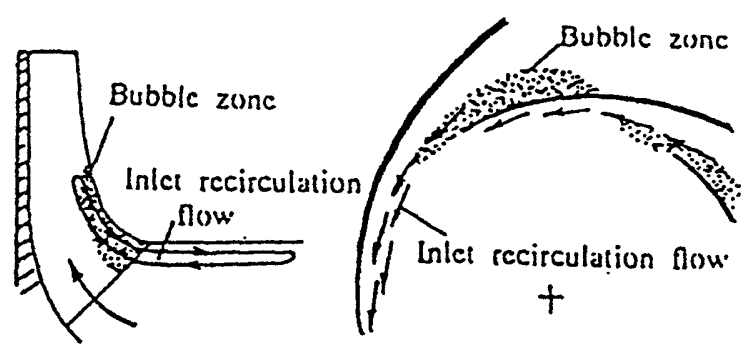

(a) Recirculation flow region and bubble zone

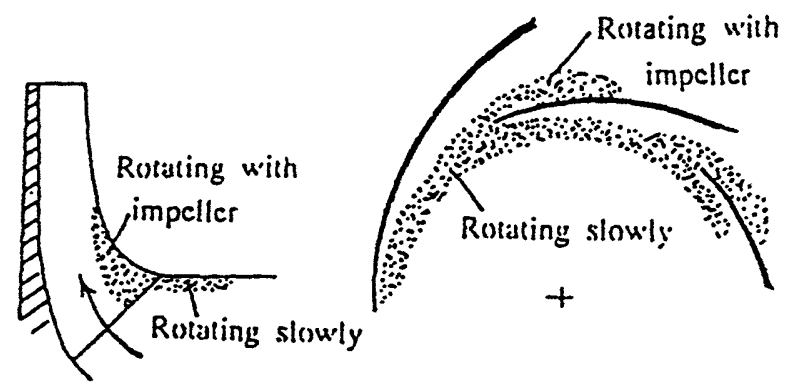

(b) Just beforc the bubble dicharging

Figure 12. Sketch of bubble behavior and recirculation flow at pump entrance 
blade loading, which results in positive slope of head-capacity curve. On the contrary in a closed type impeller the recirculation flow might form a steadily staying bubble zone which coexists stably with a recirculation flow.

From the above-described considerations the disappearance of the recirculation flow due to the growth of bubble zone might be responsible for the present oscillation, and such difference in the bubble behavior between a semi-open type impeller and a closed type could be expressed by the difference in $\alpha$ and $M_{b}$ in Eq. (8).

When a pump is operated near the shut-off point, the impeller inlet recirculation flow develops to be so strong and large-sized that the growth of bubble zone cannot cause the recirculation flow to disappear. The pumping system thus does not come into a dynamic instability but rapidly come into a static instability, resulting in a large head-drop.

\section{Conclusions}

A low frequency oscillation caused by a semi-open impeller at 'Jow discharge operation in gas-liquid flow is studied. The characteristics of the oscillation are determined experimentally and its mechanism is studied theoretically by use of a linear stability analysis.

The measurements revealed that the head drop is caused by the accumulation of bubbles along a blade pressure side around the leading edge. Theoretical considerations suggest that the bubble accumulation in blade-to-blade channels could be responsible for the present oscillation. The theoretical model provides one possible explanation as for the excitation of the oscillation, that it is caused by dynamic unbalance between the head drop rate due to bubble accumulation and the system resistance rate under the influence of flow interference between the bubble accumulating zone and the recirculation flow.

The reason why the present phenomena occurs only in a semi-open type impeller might be that the recirculation flow at impeller inlet is so unstable that the growth of bubble accumulating zone near the blade leading edge suppresses the recirculation flow and makes it disappear, which rapidly causes a large recirculation flow.

\section{Acknowledgements}

The present authors would like to express their sincere gratitude to Dr. K. Yamamoto, Dr. M. Aoki and Mr. I. Ichiki of Ebara Research Co. Ltd. for their valuable suggestions and financial support in the present study.

\section{References}

(1) Y'amamoto, K., Instability in a Cavitating Centrifugal Pump, JSME International Jr., Ser. 2, Vol. 34, No. 1(1991), pp. 9-17.

(2) Brennen, C. and Acosta, A. J., The Dynamic Transfer Function for a Cavitating Inducer, Truns. AS.WE, Jr. Fluid Dynamics, Vol. 98, No. 2(1976), pp. 182-191.

(3) Kaneko, M. and Ohashi, H., , Self-Excited Oscillations of a Centrifugal Pump System under Air/Water Two Phase Flow Condition, Proc. LAHR Symp.(Amsterdam), Vol. 2 (1982), Paper No. 36.

(4) Ichiki l., Airlocking Phenomena at Partial Load Operation in Centrifugal Pump, Ebara Research Report (in Japanese), Vol.151(1991-4), p. 24.

(5) Gleitzer, E. M., The Stability of Pumping Systems, Trans. ASME, Jr. Fluid Engineering, Vol. 103, No. 2(1981), pp. 193-242. 